
internationales

vol. 32 - $n^{\circ} 1 \mid 2016$

Les migrations des Roms roumains en Europe

\title{
Mouvements migratoires et circulation des Roms roumains en Europe
}

Migratory Movements and Circulation of Romanian Roma in Europe

Movimientos migratorios y circulación de los roma rumanos en Europa

\section{Alain Reyniers}

\section{(2) OpenEdition}

\section{Journals}

Édition électronique

URL : https://journals.openedition.org/remi/7618

DOI : $10.4000 /$ remi.7618

ISSN : $1777-5418$

Éditeur

Université de Poitiers

Édition imprimée

Date de publication : 1 mars 2016

Pagination : 19-34

ISBN : 979-10-90426-27-6

ISSN : 0765-0752

Référence électronique

Alain Reyniers, " Mouvements migratoires et circulation des Roms roumains en Europe », Revue européenne des migrations internationales [En ligne], vol. 32 - n¹ | 2016, mis en ligne le 01 mars 2018, consulté le 14 avril 2022. URL : http://journals.openedition.org/remi/7618 ; DOI : https://doi.org/ 10.4000/remi.7618 


\section{Mouvements migratoires et circulation des Roms roumains en Europe}

\section{Alain Reyniers ${ }^{1}$}

La fin du régime de Ceausescu en novembre 1989 a précipité une émigration importante de ressortissants roumains, notamment des Roms, vers l'Europe occidentale. À cette époque, I'arrivée de ces migrants à l'Ouest fut surprenante et décrite comme une invasion massive, une course éperdue vers un paradis occidental, une déferlante humaine sur un Eldorado ahuri, une fuite vers la liberté. Depuis lors, la Roumanie s'est progressivement transformée et rapprochée des États de l'Ouest. Pourtant, les mouvements migratoires n'ont pas cessé, entraînant toujours les mêmes réactions de stupeur, de lassitude et de rejet, donnant ainsi l'impression d'être face à un phénomène qui, par sa permanence, s'avère atemporel et non évolutif. Cette dernière semble, il est vrai, amplement étayée, tant par les médias qui, tout pays confondu, mettent toujours I'accent sur le déracinement des Roms, la misère de leur habitat, la dégradation de leurs conditions de vie, leur propension à vivre d'expédients et, pour tout dire, sur le dos des populations installées, que par les pouvoirs publics dont l'action répressive freine un peu partout leur établissement et les renvoie " chez eux ". Mais, peut-on pour autant en rester là et accepter cette vision des choses comme un pis aller, s'interdisant ainsi toute démarche explicative en se limitant au seul rejet ou à I'acceptation humaniste?

\section{Les Roms et la mobilité}

Cet article a pour objectif de faire le point sur ces mouvements de population romani au départ de la Roumanie et cerner, vingt-cinq ans après les premiers déploiements et l'engagement du pays sur les voies de l'économie libérale et de la démocratie, leurs configurations et leur dynamique. Pour cela, nous dresserons un cadre chronologique intégrant l'évolution de la politique européenne à l'égard des migrants de I'Europe de I'Est. À partir de divers rapports émanant $d^{\prime}$ 'institutions internationales et d'organisations non gouvernementales (Banque Mondiale, 2005 ; Cahn et Guild, 2008 ; Cherkezova et Tomova, 2013 ; FRA, 2009 ; Tarnovschi ; 2012) dédiés à la situation des Roms, nous tenterons de cerner comment les mouvements migratoires des Roms roumains ont pu apparaître dans ce cadre chronologique. Puis, à partir de nos propres enquêtes sur le terrain

\footnotetext{
1, Anthropologue, professeur à I'Université Catholique de Louvain, directeur de la revue Études tsiganes, Ruelle de la Lanterne magique, 14 bte L2.03.02, 1348 Louvain-la-Neuve, Belgique; alain.reyniers@uclouvain.be
} 
et d'autres travaux ethnographiques, nous tenterons enfin de nous mettre au plus proche des Roms engagés dans ces déplacements.

Au préalable, il convient de donner une série de précisions sur ce que l'on entend par le terme "Rom " et sur les sources sollicitées pour cet article. Qui sont les Roms ? L'appellation est couramment utilisée aujourd'hui et donne l'impression que l'on a affaire à une entité bien constituée. Le terme lui-même est tiré de la romani shib (la langue parlée par les Roms), et revêt trois significations. Au sens strict, il signifie l'homme marié. Il désigne également un groupe ethnique installé principalement au centre et à l'Est de l'Europe, notamment en Roumanie. Depuis une vingtaine d'années, il est aussi utilisé en lieu et place du terme "tsigane ", devenu synonyme d'asocial dans nombre de pays de l'Est, pour désigner l'ensemble des populations appelées également Gitans, Manouches, Sinti, voire Gens du voyage, Travellers, etc. Les Roms se distinguent des Gadjé (les non-Roms, les " gens attachés à la terre ", les " paysans "). Pour les linguistes, la langue des Roms atteste d'une origine incontestablement indienne et seuls ses locuteurs sont Roms. Nombre d'activistes et de politiciens roms reprennent cela à leur compte, insistant parfois aussi sur l'idée qu'ils appartiennent à une diaspora. Certains vilipendent l'idée que les Roms sont des nomades ou, à tout le moins, des gens rompus à la mobilité géographique. D'autres font la différence entre Roms traditionnels dont les traits culturels sont liés, entre autres, à la pratique de l'itinérance et Roms roumanisés intégrés à la population sédentaire et ayant adopté les manières des Gadjé. Les rapports qui émanent des institutions internationales dépeignent plutôt une population particulièrement précarisée et rejetée qui forme la plus grande minorité européenne. Les anthropologues, quant à eux, mettent en avant, tant la diversité de cette population que son enracinement social et économique dans les régions fréquentées. Ils insistent sur le fait que les configurations sociales des Roms reposent davantage sur des réseaux d'interconnaissance très divers plutôt que sur le seul critère ethnique.

Les divers rapports et autres documents établis sur les Roms ne font pas toujours état de ces singularités. II faut avoir cela en tête avant de s'y référer. Pour notre part, nous adoptons l'approche des anthropologues qui correspond à ce que nous avons cerné sur le terrain, mais nous interrogeons aussi la littérature consacrée aux migrations des Roms sans plus de précision. Les données contenues dans les sources écrites disponibles ne peuvent être acceptées sans prudence. Les chiffres, notamment, ne portent souvent que sur des Roms identifiables à partir de certains critères précis, tels I'habitat précaire et une situation de grande pauvreté. Ainsi, en France, par exemple, certaines statistiques ne traitent que des Roms en bidonvilles. Une fois ceux-ci sortis de ce type d'habitat, ils n'existent plus comme tels. Souvent, c'est l'état de précarité apparent qui sert de critère d'identification, en Roumanie comme dans les pays qui les reçoivent. Les extrapolations à partir de ces données doivent donc être faites avec prudence. Toutefois, elles donnent des ordres de grandeur et des tendances que nous considérons comme plausibles. La dimension anthropologique de notre propos repose avant tout sur un long terrain émaillé de plusieurs missions en Roumanie réalisées surtout entre 1993 et 2000 ainsi que sur la fréquentation régulière de Roms migrants rencontrés aussi bien en France qu'en Belgique et qui font état d'une expérience diversifiée de la migration. Elle se nourrit aussi de l'apport de plusieurs recherches doctorales dans lesquelles nous avons été impliqués. 


\section{Contexte historique}

Contrairement à une idée trop répandue, les Roms ne constituent ni un conglomérat d'individus girouettes bougeant au gré du hasard, ni un peuple de nomades définitivement réprouvés. Selon les travaux des historiens spécialistes de la question des Roms, leurs déplacements sont corrélés à leur culture autant qu'au développement des sociétés dans lesquelles ils se sont insérés². Les mouvements migratoires récents ${ }^{3} s^{\prime}$ intègrent dans ce qu'il est convenu $\mathrm{d}^{\prime}$ appeler une troisième migration (la première étant liée au départ de I'Inde au Xle siècle et à une première installation dans I'Empire byzantin au XIle siècle puis, en Europe, entre le XIVe siècle et la fin du XVe siècle, la deuxième s'étant déroulée dans la seconde moitié du XIXe siècle et au début du XXe siècle). Cette nouvelle migration a démarré au lendemain de la Seconde Guerre mondiale et se déroule encore sous nos yeux. Plusieurs phases ont pu être décrites : arrivée de réfugiés dans les quinze années qui suivent le conflit mondial, venue de travailleurs immigrés yougoslaves dans les années 1960 et 1970, apparition de candidats réfugiés originaires des Balkans dans le courant des années 1980 avec pour toile de fond la déflagration de laYougoslavie, mouvements contemporains liés à la désintégration des régimes communistes et à la transition vers des régimes libéraux. Toujours présentés comme étant liés à un flux univoque de I'Est vers l'Ouest, ces mouvements sont plus complexes. Des Roms se déplacent entre la Roumanie et la Pologne, entre la Roumanie et la Hongrie, entre la Slovaquie et la République tchèque, entre la Roumanie, I'Albanie ou la Bulgarie, la Grèce et la Turquie. On assiste également à une reprise très importante du voyage chez les Roms de I'ancienne URSS. À regarder cela à l'échelle continentale, la mobilité semble bien, plus que jamais, une caractéristique essentielle des populations romanis. De là à ne voir celles-ci qu'à travers le prisme déformant de l'image romantique d'une population errante, sans toit ni territoire, il n'y a qu'un pas trop vite franchi.

Les déplacements actuels des Roms roumains hors de leur pays d'origine ne constituent donc qu'une partie des mouvements qui touchent de nombreux Roms installés en Europe centrale et orientale. Depuis 1990, la venue des Roms de l'Est dans les pays d'Europe occidentale est expliquée autant par l'absence de perspectives économiques dans les pays d'origine que par une exclusion sociale massive sous l'effet de poussées ultranationalistes et xénophobes ${ }^{4}$. II est vrai que depuis la chute des régimes socialistes, la situation d'une majorité de Roms s'est profondément dégradée un peu partout dans les anciens pays satellites de I'Union soviétique, laissant des centaines de milliers de gens sans emploi, sans couverture sociale, sans logements décents et à la merci de pogroms ou de ratonnades multiples. À l'époque de la guerre froide, les gens qui cherchaient refuge en Occident obtenaient assez facilement l'asile politique. Tout naturellement, c'est la demande d'asile qui a été mise en avant par les migrants roms

2 Bien que largement méconnue du grand public, I'histoire des Roms existe et est bien étayée. Voir, par exemple, Asséo (1994) et Fraser (1992).

3 Plusieurs travaux portent sur cette dernière migration, notamment Marushiakova et Popov (2010), Matras (2000) et Reyniers (1995).

4 La désagrégation des anciens États communistes s'est soldée par la fermeture des anciennes entreprises d'État, I'engagement dans les voies de l'économie de marché et nombre de conflits interethniques qui n'épargnèrent nullement les Roms. 
pour justifier leur venue en Europe occidentale durant les premières années qui suivirent l'effondrement de l'ordre communiste. Les dizaines de milliers de Roms en provenance de Roumanie, de Bulgarie, de Pologne, de la République tchèque ou de Slovaquie allaient constituer une petite partie des millions d'Européens de l'Est partis en quête de travail et de vie décente en Occident ${ }^{5}$. Mais c'est leur visibilité accrue qui les a singularisés par rapport aux autres migrants : déplacements en grandes familles, demandes d'asile politique justifiées par des atteintes aux droits de l'homme dans les pays d'origine, logements dans des campements précaires ou dans des habitations insalubres, mendicité publique, etc. Et cela a ravivé, jusqu'à l'excès sans doute, les vieux préjugés à l'égard des Bohémiens et des Romanichels, bien inscrits depuis des siècles dans les mentalités des peuples européens. Des gens instables, ayant adopté un mode de vie nomade, des étrangers aux localités traversées, vivant aux crochets, voire, aux dépens des populations sédentaires.

Depuis 1990, l'État roumain s'est engagé à améliorer la situation des Roms. Ceux-ci ont été reconnus comme minorité nationale. La législation s'est, peu à peu inspirée de la convention-cadre européenne de 1998 pour la protection des minorités nationales. Avec sept autres pays ${ }^{6}$, la Roumanie s'est engagée dans la Décennie 2005-2015 des Roms, lancée par la Banque mondiale, I'Open Society Institute de Georges Soros et I'UNDP (United Nations Development Program). Globalement, la situation des Roms en Roumanie reste pourtant précaire, voire empire pour la majorité d'entre eux (Banque mondiale, 2005). Les difficultés que rencontre un nombre important de Roms sont énormes et paraissent insolubles. La seule absence de certificats de naissance, par exemple, amène à une inexistence administrative, provoque une avalanche de problèmes administratifs et de privation de droits parmi les plus fondamentaux comme l'accès aux soins de santé, à l'éducation, au logement ou au travail (Cahn et Guild, 2008 : 60). Quelques grands programmes de développement sont bien mis en place, des initiatives privées sont développées, des actions sont entreprises pour maintenir la population romani dans le pays. Mais tout cela tarde à faire sentir ses effets et nombre de Roms vivent dans et par la migration vers I'Ouest.

\section{Les phases de la migration}

À partir de 1990, les premiers mouvements importants de Roms roumains vers l'étranger se font dans le contexte du départ des Souabes et des Saxons qui, en quelque sorte, ont ouvert la voie. Le plus souvent, ils sont justifiés par le racisme exacerbé et la violence interethnique qui touchent les Roms, tout comme par la pauvreté qui grandit dans le pays. Ces mouvements sont divers, transfrontaliers pour les uns, internationaux pour le plus grand nombre. Des Roms issus des départements à majorité hongroise se dirigent vers la Hongrie pour y travailler et y faire du commerce. Certains d'entre eux s'y installent dans la durée, d'autres développent un mouvement migratoire pendulaire entre leur

5 Au plus fort des déplacements de Roms vers l'Ouest, durant la première moitié des années 1990, ceux-ci étaient huit fois moins nombreux à migrer que l'ensemble des nonTsiganes issus des pays de l'Europe centrale et orientale (Reyniers, 1995 et 2003).

6 La République tchèque, la Hongrie, la Slovaquie (à I'époque membres de I'Union européenne), plus la Bulgarie et la Roumanie, ainsi que la Croatie, la Macédoine et la SerbieMonténégro. 
localité d'origine et la ville hongroise où ils recherchent des revenus. Des Roms de Fagaras vont en Pologne qu'ils rejoignent par l'Ukraine ou la Hongrie et la Slovaquie. D'autres, plus nombreux, venant surtout de la partie occidentale de la Transylvanie ou du Banat rejoignent l'Allemagne, la France et I'Italie. Des familles provenant de Valachie entrent à leur tour dans le mouvement. Tous ces déplacements donnent l'impression qu'ils se situent dans un mouvement de fond, univoque, sans espoir de reflux, poussant des gens désemparés vers d'hypothétiques pôles de fixation, le rejet ici poussant à aller s'établir là-bas. L'accès interdit là-bas invitant à se déplacer ailleurs. Certains, parmi ces Roms, vont obtenir une aide financière temporaire ; après bien des péripéties, d'autres finiront par obtenir un logement décent et finiront par s'implanter durablement dans l'une ou l'autre localité d'élection. La plupart, vivant d'expédients, seront longtemps ballotés d'un terrain vague à l'autre, d'un squat à l'autre. Peu à peu, une fois passé l'effet de surprise, l'octroi de l'asile politique va diminuer et nombre de Roms migrants vont être renvoyés chez eux, parfois au comptegoutte, parfois en grands groupes, parfois par avion spécialement affrété pour cela. L'année 1995 sera particulièrement marquée par l'utilisation de ces " charters de la honte ", notamment entre I'Allemagne et la Roumanie.

Dans les années qui suivent, les mouvements migratoires des Roms sont liés à des événements juridiques qui concernent aussi les autres migrants roumains. À partir du $1^{\text {er }}$ janvier 2002, les citoyens roumains qui désirent circuler moins de trois mois dans l'espace Schengen n'ont plus besoin de visas, mais ils ne reçoivent pas pour autant le droit d'y travailler. Cette mesure favorise la liberté de circulation et occasionne une arrivée nouvelle de migrants au sein de l'espace Schengen. Ceux-ci sollicitent moins la demande d'asile - qui reste encore importante - et développent des activités qui ressortent de l'économie informelle. Les déplacements des Roumains à l'étranger, tous groupes nationaux ou ethniques confondus, se banalisent et le fait d'aller travailler, même en noir, hors du pays entre dans les mœurs. Les déplacements pendulaires entre la ville ou le village d'origine et l'endroit d'Europe où I'on espère du travail ou, tout au moins, des ressources, bercent l'existence de très nombreux ressortissants roumains. Pour les Roms, les destinations préférées restent majoritairement celles qui avaient été ouvertes au début des années 1990 par des proches, des parents, des familiers.

Le cas de l'Espagne est intéressant (Gamella, 2006). Au début des années 1990, quelques centaines de Roms y arrivent, au terme d'un cheminement emprunté par bien d'autres migrants roumains, à travers la Pologne, l'Allemagne et la France ou I'Autriche, I'ex-Yougoslavie, I'Italie et la France. Au début des années 2000, un nombre plus conséquent de Roms choisissent I'Espagne comme destination et y parviennent directement, au départ de la Roumanie. Ils s'installent en Catalogne, dans la région de Madrid, en Andalousie et dans le Pays basque, là où il y a du travail. Néanmoins, ils y développent une grande mobilité intérieure, en fonction du travail, saisonnier ou temporaire qu'ils peuvent y trouver.

Au $1^{\text {er }}$ janvier 2007, en vertu d'un traité d'adhésion signé en avril 2005, la Roumanie et la Bulgarie entrent officiellement dans I'Union européenne. Cet événement, majeur sur le plan politique, a une incidence directe sur le plan de la mobilité internationale des citoyens roumains et bulgares. Sur le plan des 
principes, ceux-ci reçoivent la liberté de circuler et de s'établir dans les pays de I'Union, notamment pour y travailler, mais plusieurs États vont leur imposer une période transitoire de restrictions (prévue dans le traité pour une période de deux, cinq ou un maximum de sept ans ${ }^{7}$ ) avant que celle-ci ne soit totalement effective. Les demandes d'asile, très en vogue durant les années 1990 et la première partie de la décennie 2000 ne disparaissent pas complètement, mais constituent dorénavant la portion congrue des mobiles présentés par les Roms pour s'installer. Dorénavant, les Roms roumains, comme tous les citoyens d'un pays de I'Union n'ont plus besoin de visa et bénéficient d'une possibilité de résidence dans les autres pays de l'Union durant trois mois, le délai étant rallongé de six mois en cas de recherche d'un emploi. Les expulsions ne vont plus de soi, sauf en cas de charge pour les services sociaux, pour des faits de nuisance ou de délinquance ou pour prolongation excessive de la présence dans le pays d'accueil.

Conséquence de cette réglementation nouvelle, des Roms roumains se dirigent toujours plus nombreux vers des pays, tels I'Espagne et le Portugal, qui étaient déjà attractifs par les emplois offerts dans l'agriculture, notamment, ou apparaissent en plus grand nombre dans des pays qui ne les voyaient guère ou peu jusque-là. À partir de 2008, ils sont bien présents en Angleterre, en Irlande et en Finlande, tout comme en Suisse. Conséquence de cette poussée migratoire nouvelle : I'image de la précarité apparente d'un nombre important de Roms migrants se répand un peu partout en Europe. L'idée que ceux-ci forment un groupe parasite, fermé, séparé de la société, fait de miséreux qui mendient, volent, exploitent les enfants et ne veulent ou ne peuvent s'y intégrer se développe dans des proportions inquiétantes ${ }^{8}$. Ce regard négatif alimente le rejet à leur égard parmi les populations qui les voient venir. Dans plusieurs endroits, la peur xénophobe des habitants va aller en s'amplifiant, poussant parfois certains d'entre eux à des actes violents à leur égard (en Allemagne, en Italie, mais aussi en France, comme à Marseille en 2008).

Dans ce contexte, certains États vont prendre des mesures contre la présence des Roms migrants et pour la reconduite de ces derniers vers leur pays d'origine. Les mesures prises contre les Roms roumains (notamment la destruction des maisons et l'expulsion de leurs habitants) s'accélèrent en Italie à partir de 2007 et en 2008. Des mesures législatives sont prises à partir de mai 2008 par le gouvernement italien pour faciliter l'expulsion des étrangers en situation irrégulière (celles-ci ne sont pas prises nommément contre les Roms, mais c'est eux qu'elles touchent). Des expulsions collectives de Roms roumains ont lieu en Suisse, notamment à l'automne 2007. De façon discrète, l'Allemagne fait de même. La France durcit sa position au cours de la même période, sous la présidence de Nicolas Sarkosy, avec l'organisation de " retours humanitaires ", parfois agrémentés de mesures d'accompagnement pour permettre à leurs bénéficiaires de monter une affaire ou de développer une activité agricole dans

\footnotetext{
7 Cette période maximale de sept ans a été décidée par l'Allemagne, l'Autriche, la Belgique, le Grand-Duché de Luxembourg, I'Espagne, la France, Malte, les Pays-Bas et le Royaume-Uni. Les restrictions à I'accès au marché du travail dans ces pays vont, dans les faits, progressivement être assouplies.

8 Lire le chapitre 4 du Rapport 2015 de la Commission nationale consultative des droits de I'Homme (CNCDH), publié le vendredi 10 avril 2015, à Paris.
} 
leur pays d'origine. Entre juin 2007 et mai 2008, 8349 personnes ayant bénéficié de ce soutien auraient quitté la France, principalement pour la Roumanie ou la Bulgarie $^{9}$. Une expulsion massive d'1 millier de Roms aura lieu durant l'été 2010. En 2012, ce ne seront rien moins que 12800 ressortissants roumains et bulgares qui seront expulsés (chiffres donnés par le ministère français de l'Intérieur). Mais, I'efficacité de telles mesures est douteuse. Après avoir utilisé l'incitation financière au retour pour assurer leurs besoins quotidiens, notamment en Roumanie, plusieurs bénéficiaires seraient revenus en France, ou ailleurs en Europe occidentale. Un peu partout, des mesures de surveillance et de contrôle exacerbé des Roms sont mises en place.

Depuis le $1^{\text {er }}$ janvier 2014, fin de la période transitoire maximale des restrictions à l'accès au marché de l'emploi, la liberté de circulation et d'établissement des Roumains dans les divers pays de l'Union est la règle. Faut-il dès lors prévoir une nouvelle poussée migratoire ? Difficile à dire avec certitude. Les déplacements entre la Roumanie et les autres pays européens sont entrés dans les mœurs pour nombre de migrants. Toutefois, certains d'entre eux peuvent être tentés de parcourir de nouvelles contrées. En vingt-cinq ans, les mouvements migratoires se sont mués en déplacements plutôt bien rodés. Peut-on penser que la masse de ceux qui pouvaient tirer quelque chose de la migration s'est déjà installée soit dans un pays d'accueil, soit dans un mouvement pendulaire, avant 2014 ? À cet égard, il ne faut pas oublier que des mesures facilitant l'acquisition d'un emploi pour les migrants originaires de Roumanie et de Bulgarie ont pu être prises, notamment en Allemagne et en France, pour anticiper le choc probable d'une arrivée massive en $2014^{10}$.

\section{Diversité des Roms migrants}

L'image de la migration romani au départ de la Roumanie est celle, nous l'avons dit, de familles démunies, ballotées au gré de leur rejet par les populations installées, et vivant d'expédients, au hasard des routes empruntées, pour fuir une situation originelle désastreuse. Les premières arrivées de Roms roumains en Europe occidentale, au cours des années 1990, étaient surtout le fait de familles anciennement nomades, qui avaient été sédentarisées au cours des années 1960. Celles-ci ont pu accréditer cette image par leur pratique de la mendicité et leur apparente précarité (Tesăr, 2011). Mais, c'était là, la manifestation d'une activité économique plutôt bien rodée. Insensiblement, notamment au début des années 2000, des familles beaucoup plus démunies que les précédentes se sont elles aussi engagées dans la migration, sans disposer d'atouts économiques (survie au jour le jour, en disposant d'un peu d'argent de façon totalement aléatoire, souvent endettées, arrivant à peine à se nourrir, ne pouvant faire front aux dépenses liées à la santé ou à l'acquisition d'un logement salubre), ou intellectuels (difficulté à apprendre une langue étrangère, absence de formation et de réactivité, méconnaissance des droits des migrants et de la

\footnotetext{
9 Source : Romeurope et documentation personnelle.
}

10 Ainsi, pour travailler en France en 2007, les Roumains et Bulgares doivent obtenir un titre de séjour, une autorisation de travail et leur employeur doit s'acquitter d'une taxe, variable en fonction du salaire. Mais ces mesures sont progressivement assouplies et, en 2012, 300 professions qui connaissent des difficultés de recrutement deviennent accessibles à ces migrants. 
législation européenne, etc.) suffisants pour tirer parti de la mobilité. Dans le malheur, certaines familles maintiennent parfois, faute de mieux, une économie alimentée à la charité publique ${ }^{11}$. Aujourd'hui encore, il n'est pas rare de voir des Roms particulièrement démunis fouiller les poubelles des villes occidentales, à la recherche de quelque nourriture.

En fait, les situations sont beaucoup plus complexes et sont le reflet de la diversité romani ${ }^{12}$. Les Roms migrants originaires de Roumanie se distinguent autant par leur appartenance ethnique, que par leur région ou leur localité d'origine. Si certains d'entre eux arborent une manière d'être habillés qui les rend très reconnaissables, $d$ 'autres ne révèlent pas toujours leur origine ethnique. Des groupes, des familles, des couples mixtes, urbains ou ruraux, modernes ou traditionnels, des explorateurs de terres nouvelles, des entrepreneurs audacieux, de pauvres ères n'ayant que peu d'atouts en main, sont engagés dans la migration. II y a, parmi eux, des gens n'ayant d'autres projets que de faire un peu d'argent pour se bâtir une maison au pays et monter une affaire, des gens poussés par l'accès plus probe aux soins de santé et par la volonté d'assurer l'éducation de leurs enfants. On retrouve des familles séparées, disloquées ou maintenant une cohérence interne forte.

Sous l'appellation globale de Roms migrants (souvent vus comme pauvres), se retrouvent en fait des personnes bien différentes, ayant leurs propres projets migratoires, leurs désirs d'une vie meilleure, et développant leurs propres dynamiques économiques et sociales dans un contexte souvent difficile. Des migrants qui ne partagent pas toujours les mêmes lieux de chute et ne sont pas non plus toujours portés à se sentir solidaires et à s'entraider. Des individus souvent peu formés, mais avec de l'expérience et, notamment chez les anciens nomades, des capacités économiques à l'adaptation et à tirer parti de ce qui peut être ramassé. L'ensemble regroupe plusieurs dizaines de milliers de personnes ${ }^{13}$. II ne concernerait pour autant qu'une minorité au sein de la population romani roumaine. S'il faut en croire certaines études (Rapport Soros, $2011^{14}$ ), moins d'un tiers des Roms du pays seraient tentés par l'expérience migratoire en Europe occidentale, voire, ajoutons-le, plus loin au Canada, aux États-Unis ou en Australie.

\section{Mouvements, ancrage et réseaux}

II faut de l'argent pour s'engager dans la migration. Les uns en possèdent, d'autres vendent leurs biens ou s'endettent. De toute façon, le coût du projet migratoire est incontestable. Souvent, les emplois recherchés sont précaires, dans la construction, les travaux publics, les activités agricoles saisonnières, les

11 Voir à ce propos le témoignage de Loredana Costea dans Fierens (2012: 50).

12 Un exemple de cette diversité est donné pour la ville de Bruxelles dans Geurts (2014).

13 À noter la singularité des femmes dans ce cadre-là : les aptitudes dont elles font la preuve sont le plus souvent dépendantes de leur groupe d'appartenance. Elles vivent plutôt à la remorque du mari dans les groupes traditionnels, aptes à saisir davantage les opportunités qui s'offrent à elles dans les familles plus scolarisées.

14 Selon ce rapport, les principales destinations espérées par les Roms en 2011, seraient dans I'ordre, I'Espagne, I'Italie et la France. Le niveau d'éducation de ceux qui tentent leur chance en Espagne serait plus élevé. Les raisons des départs seraient principalement liées à la recherche d'un travail et à l'augmentation du niveau de vie. 
entreprises de nettoyage, le gardiennage, la récupération. On note aussi la vente ambulante ou à la sauvette, la musique en rue ou dans le métro, la mendicité, plus rarement la prostitution. La recherche d'une aide sociale est également motif de déplacement. Parfois, des familles anciennement implantées emploient des migrants plus récents. Lorsque c'est possible, le travail indépendant, légal ou informel, est recherché. Pour la plupart, les activités de juste subsistance sont maintenues tant que la situation reste précaire. Puis, insensiblement, lorsque les perspectives d'une installation prolongée se font jour, notamment sur le plan réglementaire, alors, la recherche d'un travail plus régulier apparaît. Certains $s^{\prime}$ implantent définitivement dans le pays $d^{\prime}$ accueil ${ }^{15}$. L'installation se concrétise de temps en temps par l'achat d'un bien immobilier. La maison éventuellement laissée en Roumanie devient maison de vacances. Dans certains cas, elle est revendue, remplacée par une habitation mieux située, plus imposante, manifestation de la réussite économique.

Le succès de l'expérience migratoire est parfois matérialisé par la construction de palais monumentaux en Roumanie. Mais ceux-ci relèvent en partie d'une surenchère entre familles en quête de prestige plutôt que le fruit d'une réelle opération économique. Dans les villages, les Roms quittent les quartiers périphériques au profit du centre de la localité en acquérant ou construisant des immeubles de meilleure qualité que ceux qu'ils possédaient avant leur départ. II faut noter aussi, avec le retour au pays, l'émergence d'entrepreneurs qui s'insèrent tantôt dans l'économie locale, comme transporteurs, fabricants d'objets divers, soldeurs sur les marchés ou maintiennent un lien avec les pays qu'ils ont fréquentés, en mettant sur pied qui une entreprise de transport routier, qui une entreprise de construction. Indéniablement, ils sont, les uns et les autres, des acteurs de la circulation des richesses dans l'espace européen et en Roumanie même.

La plupart des Roms migrants cherchent à aller vers une destination bien précise et tentent de s'y établir. Mais, pour y parvenir, les parcours, là encore, sont divers. Certaines familles vont d'un pays à l'autre, par exemple en Turquie ou en Hongrie, puis en Allemagne ou en France, avant de partir vers l'Espagne. Après une première expérience migratoire au début des années 1990, par exemple vers l'Allemagne, des Roms tentent dix ans plus tard une expérience nouvelle vers un autre pays, en profitant de la suppression des visas. Puis, ils s'engagent dans un mouvement pendulaire, dans l'exploration d'une nouvelle région, s'insèrent dans de nouveaux réseaux de relation, la plupart du temps gardant toutefois un lien avec le pays d'origine. Ainsi verra-t-on des Roms de Beius migrer, soit vers Bruxelles, soit vers Marseille. Mais, à partir de Bruxelles, certains d'entre eux s'établissent tôt ou tard auprès des leurs déjà présents dans la ville phocéenne.

\footnotetext{
15 Sur le plan de l'habitat, la diversité est également de règle. Les expulsions, les destructions de bidonvilles ne sont que la partie émergée de formes d'installation plus complexes, loin des seuls squats et bidonvilles auxquels la situation française nous a habitués. En Angleterre, en Belgique, en Allemagne, les Roms roumains logent parfois en squats, mais on les trouve plus souvent dans des appartements ou des petites maisons modestes, souvent louées à un prix prohibitif. Parfois, leur hébergement se fait dans des dispositifs dédiés, comme les villages d'insertion en France ou les campi nomadi en Italie. Parfois, le logement se réduit à un baraquement insalubre. En Espagne, on les retrouve aussi dans des campings cars et des caravanes lors des travaux agricoles saisonniers.
} 
Ou encore deux beaux-frères quittent leur village, I'un pour Dublin, l'autre pour Helsinki ; ce dernier décidant de rejoindre I'Irlande lui aussi lorsqu'il aura réuni assez d'argent en Finlande. Des cousins ayant quitté Arad, I'un pour Cologne, l'autre pour Marseille et un troisième pour Bruxelles se retrouvent à Bruxelles à l'occasion d'un mariage et l'un d'eux décide d'y rester. Les migrants deviennent des immigrés établis dans un pays d'accueil. Leurs enfants ne subissant plus de récriminations ou de vexations à l'école, l'accès à un logement décent et aux soins de santé étant assurés, le travail enfin à portée de main, certains n'ont plus envie de retourner au pays. Dans certains cas, les déploiements des parents et des amis hors de Roumanie ont poussé les gens à une dispersion telle que les liens avec le lieu de départ se sont progressivement atténués, pour être remplacés par des déplacements plus ou moins réguliers entre les nouveaux établissements en Occident. Le retour en Roumanie n'est plus à l'ordre du jour, mais un lien, fût-il symbolique, peut être entretenu avec le pays d'origine.

Plusieurs groupes de Roms roumains investis dans la migration finissent par s'ancrer dans une ville de l'Ouest sans pour autant rompre avec la ville ou le village d'origine. S'ensuit dès lors l'émergence d'un double ancrage territorial, social et économique avec des mouvements pendulaires entre les deux pôles. Et, là encore, les configurations sont diverses. Les uns alternent des séjours en hiver et au printemps, dans le pays d'accueil, retournent en été au village. D'autres font des allers retours plus fréquents ou, au contraire, séjournent plus longtemps hors de la Roumanie. Ces déplacements dépendent beaucoup des activités pratiquées et des revenus acquis.

Certains de ces mouvements sont très révélateurs de la situation économique des pays fréquentés ou simplement traversés. Durant la seconde moitié des années 2000, de nombreux Roms roumains cherchant à travailler dans l'agriculture ont migré vers la Grèce ou l'Espagne avant de repartir vers d'autres pays comme la France ou I'Italie, traduisant ainsi par leurs déplacements, I'attraction économique temporaire de certains pays qui seront ensuite délaissés pour d'autres lorsque survient une période de crise. Ce même type de déplacement, ici à l'échelle européenne, se retrouve aussi au sein d'un même pays (arrivée à Lyon, déplacement vers Saint-Étienne, départ ensuite vers Marseille) ou entre deux pays limitrophes (arrivée à Lyon, déplacement vers Paris, départ pour Bruxelles; passage de la Belgique à l'Angleterre).

Les Roms sont, pour la plupart, insérés dans des réseaux familiaux et de voisinage très flexibles, au sein desquels ils circulent habituellement. Ces réseaux de parenté et de sociabilité sont très fortement valorisés en migration. Mués en individus mobiles, les Roms parcourent les routes d'Europe en microbus ou en voitures privées et développent (ou retrouvent) une sociabilité du voyage articulée aux lieux de halte autoroutiers, aux croisements d'itinéraires progressivement bien tracés ${ }^{16}$, sur les lieux de résidence et dans les villes fréquentées. Les groupes familiaux se composent et se recomposent au sein de ces réseaux. Des alliances matrimoniales nouvelles peuvent voir le jour. Mais,

16 Ce phénomène devient bien étudié et des données ethnographiques sont disponibles ou le seront bientôt. Signalons à ce propos les thèses de Dion et de Benarrosh-Orsoni défendues en 2015. 
la propension à rester entre personnes du même groupe est particulièrement forte. Les gens qui se reconnaissent la même pratique d'un dialecte, les mêmes valeurs de référence, les mêmes origines sociales ou géographiques restent entre eux et ne se montrent pas toujours enclins à soutenir ceux qui ne sont pas du même monde qu'eux.

La religion, tout particulièrement le pentecôtisme, opère comme cadre régulateur de l'immigration. Des Églises existent dans les localités de départ. Elles se reconstituent dans les pays d'accueil, le plus souvent en fonction du groupe d'appartenance, du lieu d'origine. D'où leur contribution au renforcement d'une identité collective et des liens sociaux organisés en réseaux. Les pasteurs animent une vie sociale, spirituelle et aussi politique avec les Gadjé. Cela étant, les difficultés interculturelles sont elles-mêmes légions. En tout premier lieu, le système de solidarité interne reste limité à chaque réseau et $\mathrm{s}^{\prime} y$ renégocie sans cesse. Pas d'entraide automatique donc entre des Roms roumains et des Roms de Serbie ou de Bulgarie, méfiance entre des Roms issus de régions roumaines différentes. D'une manière générale, l'ignorance entre les Roms établis depuis longtemps à l'Ouest et ceux qui viennent actuellement de I'Est est réciproque et radicale.

\section{Des Roms migrants parmi d'autres}

L'exemple suivant, centré sur une famille originaire d'Arad, permettra de cerner concrètement le jeu des divers paramètres de la migration. Les premiers membres de la famille qui émigrèrent vers l'Europe occidentale arrivèrent à Bruxelles en 1996. Ils furent rapidement rejoints par des parents proches puis, insensiblement, par des cousins plus éloignés en provenance de Timisoara. Tous tentèrent d'obtenir l'asile politique. Bénéficiant de contacts au sein d'associations de défense des Roms, mais aussi grâce à leur propre entregent, ils ne tardèrent pas à occuper une position de leaders parmi les Roms migrants originaires de Roumanie et installés dans la capitale belge. Des contacts furent noués avec les parents récemment établis en Allemagne et en France. Tout un réseau d'échanges et de déplacements fut organisé entre Bruxelles, Marseille et Cologne. Avant même que la Roumanie ne fasse son entrée dans I'Union européenne, ces Roms initiaient un mouvement pendulaire entre l'Est et l'Ouest, alimentant tout un commerce de récupération et renforçant les liens économiques par des relations matrimoniales entre enfants de migrants et gens restés au pays. Mais, le caractère aléatoire des ressources liées à quelques allocations, divers expédients et le ramassage d'objets usagers d'une part, les rivalités économiques d'autre part, poussèrent un certain nombre de parents à se dissocier du groupe bruxellois et à rejoindre Marseille après un bref passage par Lyon. Là, de squat en squat, en combinant les ressources tirées de la mendicité des femmes à celles de la récupération, en cherchant aussi, comme à Bruxelles, à établir des liens avec les organisations qui aident les Roms, ils mirent en place un système similaire d'envoi de pièces détachées tirées de la récupération vers l'Europe de l'Est, la Roumanie bien sûr, mais également la Bulgarie et la Pologne.

Aujourd'hui, à Bruxelles et à Marseille, les membres de la famille vivent plutôt chichement. Mais, ils ont su reconstituer un espace de vie romani dans lequel circulent des gens établis aussi bien à l'Est qu'à l'Ouest de l'Europe, au 
Nord ou au Sud du continent. Un certain nombre d'entre eux ne cherchent plus à retourner en Roumanie et deviennent les pôles de référence autour desquels gravitent ceux qui tournent davantage. Les liens avec les Gadjé sont ténus. Quelques " amis desTsiganes ", desTurcs à Bruxelles, des Algériens à Marseille, des voisins, épiciers, membres d'associations culturelles d'immigrés. D'autres liens existent bien sûr, plus lâches quoique quotidiens avec les clients, tous ceux que l'on démarche ou que l'on sollicite au cours de la journée. La précarité apparente, la quête hasardeuse des revenus, les manières d'aborder les gens dans la rue, tout cela suscite la crainte et ne pousse guère les Gadjé à se représenter les Roms autrement que comme des marginaux. Mais ces derniers vivent dans un espace social et familial fort, qui leur permet d'échapper au malheur de la solitude et leur offre un accès au partage de ressources aléatoires. Ces Roms dépendent des Gadjé pour vivre - ils sont le premier maillon d'un réseau international de ramassage et de recyclage -, mais ils restent maîtres d'un univers fait d'échanges matrimoniaux, de congratulations réciproques, d'entraide, de conflits, de joies, de peines et de soucis partagés.

L'évocation de ce parcours familial n'épuise pas, bien sûr, la grande diversité des situations économiques et sociales vécues par les Roms migrants. Un peu partout en Europe, il y a des Roms réduits à la misère, qui vivent dans des zones délaissées et sans la moindre espérance (Reyniers, 2007). A contrario, il y a des familles qui brassent les affaires et exhibent leur richesse. Ici, nous avons évoqué une famille qui, malgré les déboires vécus, parvient à se relever et à vivre. Son histoire est jalonnée de périodes fastes, trop souvent insoupçonnées, et de périodes plus difficiles. Le déroulement des activités économiques dans un environnement qui lui est parfois hostile est lié à des processus de fusion et de scission entre les diverses composantes de la famille, au terme desquelles les configurations sociales changent, mais ne se diluent pas pour disparaître inéluctablement parmi les Gadjé. Nous saisissons bien, dès lors, la profonde dépendance économique de ces Roms vis-à-vis de ces derniers. Mais nous remarquons aussi combien leur flexibilité sociale les amène à faire société et à s'affranchir des Gadjé, à rester des Roms. L'argent lui-même, gagné auprès des Gadjé, est en quelque sorte lavé de son origine (Stewart, 1987) pour affirmer I'identité romani du groupe, par l'achat d'une camionnette qui sera suivi par I'achat de camionnettes similaires chez les parents, par diverses agapes entre familiers, par la participation à des événements religieux qui jettent des ponts entre les Roms de mêmes groupes ou de groupes différents. Inscrits dans des lignées multiséculaires, ces Roms se réinventent comme groupe autonome, de génération en génération, dans et avec une société globale qui se modifie.

\section{Conclusion : un état des lieux en demi-teinte}

Il faudra peut-être encore un certain temps avant de pouvoir dresser un bilan circonstancié des vingt-cinq années de mobilité romani que nous venons de passer en revue. Les déplacements hors de Roumanie sont incontestables et récurrents sur toute la période examinée. Si nous avons utilisé le terme migration pour les désigner, certains observateurs hésitent à l'employer. La majorité des Roms n'a pas quitté la Roumanie, il est vrai. Ceux qui sont partis ne cultivent pas non plus une stratégie univoque de déploiement et d'installation. II y a des départs, des retours et des va-et-vient multiples. Peut-on alors parler 
de circulation (des gens de peu qui expérimenteraient l'Europe à leur manière) ? Ne doit-on y voir que de la gesticulation sans grande conséquence? Des mouvements ponctuels de population ? Un peu de tout cela?

Ces mouvements migratoires (appelons-les ainsi) ne sont pas totalement visibles. Mais ceux qui frappent les observateurs, les politiciens et le grand public donnent des Roms l'image collective singulière d'un groupe démuni pratiquant des activités à la limite du licite. La qualification de Roms errants est probablement moins fréquente qu'il y a encore quinze ans. Aujourd'hui, les médias et les pouvoirs publics parlent plutôt de Roms roumains, de Roms en bidonvilles, de Roms en situation précaire, de Roumains renvoyés vers leur pays d'origine, désignant par là un phénomène réduit à quelques-unes de ses apparences, mais banalisé dans la quotidienneté. Ces représentations restent très négatives et globalisent les Roms comme un groupe pauvre, déterritorialisé. Insidieusement, une frontière ethnique basée sur l'ethnicisation de la pauvreté vient diviser les peuples européens et pousse les Roms dans une ghettoïsation symbolique qui accompagne bien souvent leur mise à l'écart explicite.

Les Roms eux-mêmes ne réagissent pas à cela par un surcroît d'identification collective qui dépasserait leurs propres clivages internes. La conscience de faire partie d'un ensemble transnational marginalisé ne se développe qu'au niveau de certains leaders et intellectuels politisés. La majorité des Roms reste, quant à elle, particulièrement attachée à ses singularités ethniques internes qui sont, comme nous l'avons vu, entretenues, voire renforcées, dans le cadre migratoire. Cela étant, ceux qui entreprennent ces mouvements migratoires s'en tirent souvent mieux que les plus pauvres restés au pays, à qui s'adressent les aides internationales ou de grands programmes de développement. Plus que jamais, la mobilité serait-elle pour les Roms la réponse qu'ils apportent aux situations de crise qui les touchent?

En tout cas, force est de constater qu'un grand nombre de Roms migrants finissent toujours par renforcer, voire renouveler leur ancrage territorial et social dans la contrée qu'ils ont quittée et font de même, si possible, dans la contrée d'immigration avec à la clé, création de rapports nouveaux avec les Gadjé locaux. Ce faisant, ces Roms arborent une culture du lien social, qui les concerne, mais qui s'avère également participative à la culture de l'environnement gadjo, une culture axée sur l'insertion territoriale et la mobilité, qui tient compte des contraintes économiques, mais aide aussi à faire des choix dans ce domaine vital. En cela, et malgré les multiples jeux de singularité identitaire qu'ils mettent au point et renouvellent, les Roms sont aux antipodes de ce monde fermé et exclusif, négationniste de l'autre, qui leur donne bien du fil à retordre. 


\section{Références bibliographiques}

Asséo Henriette (1994) Les Tsiganes, une destinée européenne, Paris, Gallimard, $160 \mathrm{p}$.

Banque Mondiale (2005) Roma in an Expanding Europe, Breaking the Poverty Cycle, Washington, D.C., The World Bank, 270 p.

Benarrosh-Orsoni Norah (2015) Des maisonnées transnationales. Une migration rom dans ses routes, lieux et objets entre la Roumanie et la France, Thèse en ethnologie, Université de Paris Ouest-Nanterre La Défense.

Cahn Claude and Guild Elspeth (2008) Recent Migration of Roma in Europe, 10 December, OSCE High Commissioner on National Minorities, 94 p., [online] last checked on 30/06/2016. URL: http://www.osce.org/hcnm/78034?download=true

Cherkezova Stoyanka and Tomova llona (2013) An option of last resort? Migration of Roma and Non-Roma from CEE countries, Roma Inclusion Working Papers, Bratislava, UNDP, $202 \mathrm{p}$.

Dion Sébastien (2015) Un monde européen, entre changements et mobilités. Une étude ethnographique menée auprès de familles roms roumaines en contexte migratoire, entre la région historique de Crisana (Roumanie) et l'agglomération lyonnaise (France), Thèse en ethnologie, Université Catholique de Louvain, $481 \mathrm{p}$.

Fierens Jacques (Dir.) (2012) Les Roms face au droit en Belgique, Bruxelles, La Charte, 302 p.

FRA (European Union Agency for Fundamental Rights) (2009) The situation of Roma EU citizens moving to and settling in other EU member states, Luxembourg, Office for Official Publications of the European Communities [online] last checked on 30/06/2016. http://fra.europa.eu/sites/default/files/fra uploads/705-Roma_Movement_Comparative-final_en.pdf

Fraser Angus (1992) The Gypsies, Oxford and Cambridge (UK), Blackwell, 359 p.

Gamella Juan Francesco (2006) L'immigration ignorée. Les Roms roumains en Espagne, Études tsiganes, 27-28, pp. 116-154.

Geurts Koen (2014) Les Roms en action. Différents groupes et évolutions à Bruxelles. Suggestions pour le travail social, Bruxelles, Centre régional d'intégration Foyer, 208 p.

Legros Olivier et Tommaso Vitale (2011) Les migrants roms dans les villes françaises et italiennes : mobilités, régulations et marginalités, Géocarrefour, 86 (1), [en ligne] consulté le 12/01/2015. URL : http://geocarrefour.revues.org/8220

Lièvre Marion (2013) Nationalisme ethnoculturel et rapport à la culture des Roms en Roumanie postcommuniste et multiculturaliste, Thèse en ethnologie, Université de Montpellier III - Paul Valéry, 504 p.

Marushiakova Elena and Popov Vesselin (2010) Gypsy/Roma European migrations from $15^{\text {th }}$ century till nowadays, Paper presented at the International Conference - Romani Mobilities in Europe: Multidisciplinary Perspectives, 14-15 January 2010, Refugee Studies Centre, University of Oxford.

Matras Yaron (2000) Romani Migrations in the Post-Communist Era: Their Historical and Political Signifiance, Cambridge Review of International Affairs, 13 (2), pp. 32-50. 
Oliveira Martin (Dir.) (2009) Roms de Roumanie, la diversité méconnue, Études tsiganes, 38, $194 \mathrm{p}$.

Reyniers Alain (2007) Entre inexistence sociale et affirmation culturelle, le cas des Roms en Slovaquie orientale, in Vinciane Châtel Éd., L'inexistence sociale. Essai sur le déni de l'autre, Fribourg, Academic Press Fribourg et Éditions Saint Paul, pp. 115-123.

Reyniers Alain (Dir.) (2006) Migrations tsiganes, Études tsiganes, 27 et 28.

Reyniers Alain (2003) Migrations tsiganes de Roumanie, in Dana Diminescu, Visibles mais peu nombreux. Les circulations migratoires roumaines, Paris, Éditions de la maison des sciences de l'homme, pp. 51-64.

Reyniers Alain (1995) Les populations tsiganes et leurs mouvements dans les pays d'Europe centrale et orientale et vers quelques pays de I'OCDE, Paris, OCDE (Migration Internationale et Politique du Marché duTravail, Document hors-série, $\mathrm{n}^{\circ} 1$, Diffusion générale).

Stewart Michael (1987) Brothers in Song. The Persistance of (Vlach) Gypsy Identity and Community in Socialist Hungary, Theses submitted for the degree of Ph. D. at the London School of Economic and Political Science, Faculty of Economies.

Tarnovschi Daniela (Dir.) (2012) Roma from Romania, Bulgaria, Italy and Spain between social inclusion and migration, Bucharest, Soros Fundation.

Tesăr Cătălina (2011) Good "traditional" Gypsies in Romania, de-ethnicized beggars abroad, in Stefánia Toma and László Fosztó Eds., Spectrum. Cercetări sociale despre romi, Cluj, ISPMN - Kriterion. 


\section{Alain Reyniers \\ Mouvements migratoires et circulation des Roms roumains en Europe}

Cet article fait le point sur les mouvements migratoires des Roms de Roumanie et cerne, vingt-cinq ans après les premiers déploiements en Europe et l'engagement du pays sur les voies de l'économie libérale et de la démocratie, leurs configurations et leur dynamique. Depuis la chute des régimes communistes, I'arrivée des Roms en Europe occidentale est expliquée autant par l'absence de perspectives économiques dans le pays d'origine que par une exclusion sociale massive. Les premiers Roms migrants originaires de Roumanie ont justifié leur venue à l'Ouest par ces arguments et ont présenté des demandes d'asile, tout en vivant d'expédients. Avec l'instauration de l'espace Schengen, puis l'intégration de la Roumanie dans I'Union européenne, les stratégies migratoires vont se diversifier. Elles témoignent, pour la plupart, d'un ancrage territorial fort et d'une pratique de l'itinérance qui s'appuie sur des réseaux familiaux qui restent une référence.

\section{Migratory Movements and Circulation of Romanian Roma in Europe}

This article examines the migrations of Roma from Romania twenty five years after the first arrivals in Europe and the country's commitment to the ways of the market economy and democracy. The article identifies both the configuration and dynamics of the Romanian Roma. Since the fall of communist regimes, the Roma arrival in Western Europe is explained largely by the lack of economic opportunities in the country of origin and by a massive social exclusion. The first Roma migrants from Romania justified their coming to the West by these arguments and submitted applications for asylum, while hustling for a living. With the introduction of the Schengen area and the integration of Romania into the European Union, migration strategies were diversified. Most of them revealed strong social roots and a mobility based on family networks, which remained the reference point.

\section{Movimientos migratorios y circulación de los roma rumanos en Europa}

En este artículo se examina la migración de los roma rumanos y se establecen, veinticinco años después de los primeros despliegues en Europa y del compromiso del país con la economía liberal y la democracia, sus configuraciones y sus dinámicas. Desde la caída de los regímenes comunistas, la llegada de roma a Europa occidental se explica tanto por la falta de oportunidades económicas en el país de origen como por su exclusión social masiva. Los primeros migrantes roma procedentes de Rumanía justificaron su llegada a Occidente en relación a estos factores y optaron por presentar sus solicitudes de asilo, mientras vivían de su ingenio. Con la instauración de la zona Schengen y la integración de Rumanía en la Unión Europea, las estrategias de migración se diversificaron. Estas reflejaban, en su mayor parte, un fuerte arraigo y una alta movilidad habitacional, basada en las redes familiares, establecidas como puntos de referencia. 